\title{
A Multi-Variate Predictability Framework to Assess Invasive Cardiac Activity and Interactions during Atrial Fibrillation
}

\author{
Alejandro Alcaine*, Michela Masè, Member, IEEE, Alessandro Cristoforetti, Flavia Ravelli, \\ Giandomenico Nollo, Member, IEEE, Pablo Laguna, Senior Member, IEEE, \\ Juan Pablo Martínez and Luca Faes, Member, IEEE
}

\begin{abstract}
Objective: This study introduces a predictability framework based on the concept of Granger causality (GC), in order to analyze the activity and interactions between different intracardiac sites during atrial fibrillation (AF). Methods: GC-based interactions were studied using a three-electrode analysis scheme with multi-variate auto-regressive models of the involved preprocessed intracardiac signals. The method was evaluated in different scenarios covering simulations of complex atrial activity as well as endocardial signals acquired from pa-tients. Results: The results illustrate the ability of the method to determine atrial rhythm complexity and to track and map propagation during AF. Conclusion: The proposed framework provides information on the underlying activation and regular-ity, does not require activation detection or post-processing algorithms and is applicable for the analysis of any mul-ti-electrode catheter. Significance: The proposed framework can potentially help to guide catheter ablation interventions of $\mathrm{AF}$.
\end{abstract}

Index Terms - Atrial fibrillation, Bipolar electrograms, Granger causality, Multi-electrode catheters, Multi-variate au-to-regressive modelling.

Manuscript received; revised; accepted. This work was supported by personal grants to A.A. refs: BES-2011-046644 and EEBB-I-14-08406, by project TEC2013-42140-R from Ministerio de Economía y Competitividad. Also by Aragón Government (Spain) and European Social Fund (EU) through Grupo Consolidado BSICoS ref.: T96, by the Healthcare Research Implementation Program (IRCS), Provincia Autonoma di Trento, by Bruno Kessler Foundation, Italy and by CIBER de Bioingeniería, Biomateriales y Nanomedicina (CIBER-BBN) through Instituto de Salud Carlos III. The computation was performed by the ICTS "NANBIOSIS", more specifically by the High Performance Computing Unit of the CIBER-BBN at the University of Zaragoza. Asterisk indicates corresponding author

*A. Alcaine is with the BSICoS Group, Aragón Institute of Engineering Research (I3A), IIS Aragón, Universidad de Zaragoza, 50018 Zaragoza, Spain, and also with the CIBER de Bioingeniería, Biomateriales y Nanomedicina (CIBER-BBN), 28029 Madrid, Spain (e-mail: aalcaineo@unizar.es).

M. Masè A. Cristoforetti and F. Ravelli are with the Department of Physics, University of Trento, 38123 Trento, Italy (e-mail: michela.mase@unitn.it; alessandro.christoforetti@unitn.it; flavia.ravelli@unitn.it).

G. Nollo and L. Faes are with the IRCS-FBK and BIOtech, Department of Industrial Engineering, University of Trento, 38123 Trento, Italy (e-mail: nollo@science.unitn.it; faes.luca@gmail.com).

P. Laguna and J. P. Martínez are with the BSICoS Group, Aragón Institute of Engineering Research (I3A), IIS Aragón, Universidad de Zaragoza, 50018 Zaragoza, Spain, and also with the CIBER de Bioingeniería, Biomateriales y Nanomedicina (CIBER-BBN), 28029 Madrid, Spain (e-mail: laguna@unizar.es; jpmart@unizar.es).

\section{INTRODUCTION}

$\mathrm{T}$ HE increasing prevalence of atrial fibrillation (AF) turns it into an epidemiological threat [1] affecting around 1\% to $2 \%$ of the general population in US and Europe [2]. Therefore, AF is considered the most common form of sustained arrhythmia that affects the patient's quality of life [3], [4].

The early mention of AF was done by Thomas Lewis at the beginning of the 20th century [5]. Later, Moe et al. [6] proposed the first mechanism sustaining AF via multiple wavelets propagating through the atrium, which was experimentally proven by Allessie et al. [7]. More recently, the proposed mechanisms of AF include focal drivers from the pulmonary veins [8], reentries [9], and transmural connections between atrial layers [10]. However, the phenomena that initiate and perpetuate AF still remain incompletely understood [9].

Pulmonary vein isolation is the recommended ablation approach for AF treatment during the first expression of the disease and ineffectiveness of antiarrhythmic drug therapy [2]. During ablation interventions, invasive intracardiac electrogram (EGM) signals are acquired from different atrial sites using multi-electrode catheters. Point-by-point mapping using an electroanatomical mapping system can be useful for this purpose. However, in the case of complex arrhythmias, like $\mathrm{AF}$, simultaneous mapping is desirable [9].

Studying intra- and inter-relationships between these multiple atrial signals can provide insights on the underlying fibrillation process. Usually, pulmonary vein isolation is complemented with targeting complex fractionated atrial sites [11], [12], atrial sites with higher dominant frequency [13] or lower organization [14]. Therefore, multiple EGM signal processing techniques have been proposed to provide tools that may help physicians to quantify and visualize these data in order to better guide the ablation procedure [15]. Efforts are concentrated in assessing activation times [16]-[20], signal regularity and/or organization in both time or frequency domains [14], [21]-[25], combined rate and regularity measures [26], and phase maps [27]. Possible limitations of most of these techniques are that they depend on the accuracy in the detection of atrial activations, and/or do not explore the spatiotemporal interactions of the activity measured by the 
catheter electrodes.

Assessing the information flow and causal relations between different atrial sites has been proposed to quantify signal interactions using parametric models represented either in the frequency [28], [29] or time domains [30]-[33]. In this work, we propose a multi-variate predictability framework, based on the concept of Granger causality (GC) [34], which extends the possibilities of previous approaches. Causal interactions (in the sense of the GC) between different atrial sites are analyzed during different rhythms by considering the EGM signals as stochastic processes, which interact with the neighbouring atrial sites by means of an information exchange driven by the atrial activity. A set of predictability measures is defined from the residual variances of linear predictions performed in the frame of multi-variate auto-regressive (MVAR) modelling of the involved pre-processed EGM signals. Evaluation was done using simulations and clinical mapping data in order to cover the most common situations found during electrophysiological studies or ablation interventions.

The framework provides measures of the regularity of individual EGMs, as well as of the connectivity between a target and its two neighbouring atrial sites. These measures are applicable to any multi-electrode catheter, and can be computed without activation detection or post-processing steps. Therefore, the proposed framework provides a tool for the analysis of the atrial activity, performed from single- to multi-electrode points of view, which is based solely on GC definitions

This paper is organized as follows: Section II introduces the different datasets used to evaluate the proposed methodology. Section III introduces the complete framework and definitions. Section IV shows the results obtained on the different datasets and Sections V and VI present the discussion and conclusions of this work, respectively.

\section{MATERIALS \\ A. Computer simulations}

Simulation data were generated using the Courtemanche-Ramírez-Nattel ionic model [35]. This ionic model runs over a simplified human atrial geometry represented as a monolayer sphere with $6 \mathrm{~cm}$ diameter, discretized into a triangular mesh of approximately 125.000 nodes and spatial resolution of about $300 \mu \mathrm{m}$.

Bipolar EGMs were obtained using the current source approximation [36] on virtual spherical electrodes of $0.25 \mathrm{~mm}$ diameter located at $0.5 \mathrm{~mm}$ distance from the simulated anatomy. Recording electrodes were distributed over the simulated anatomy mimicking a basket catheter with eighteen regularly spaced splines ( $\pi / 9$ rad angular distance), each composed by eight regularly spaced bipoles with $2 \mathrm{~mm}$ inter-electrode distance, as illustrated in Fig. 1(a). Synthetic EGM signals of $10 \mathrm{~s}$ length were derived from each simulation with a sampling frequency of $1 \mathrm{kHz}$.

Different activity pattern scenarios were simulated in this configuration in order to cover the most common propagation patterns: a)

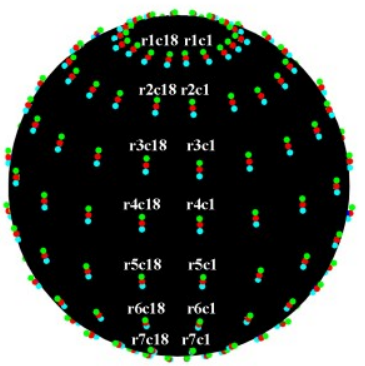

c)

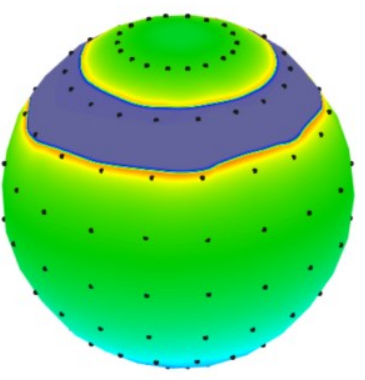

e)

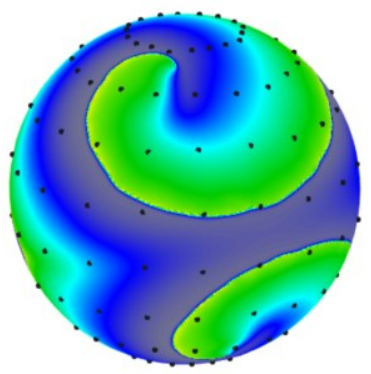

g)

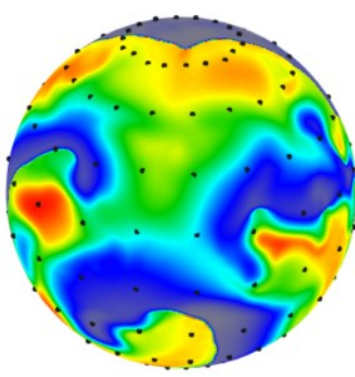

b)

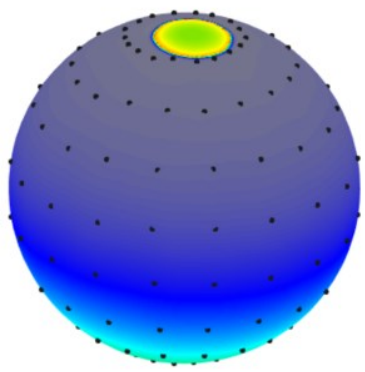

d)

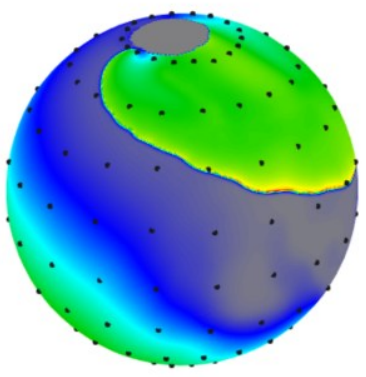

f)

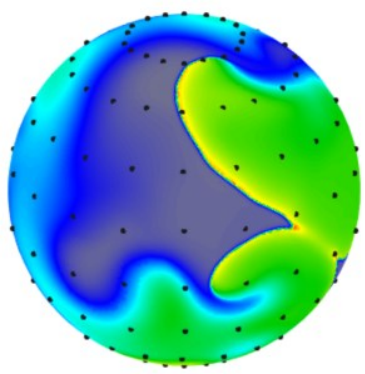

h)

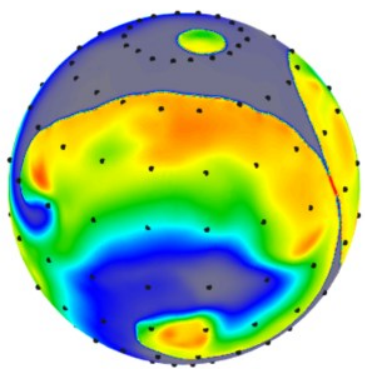

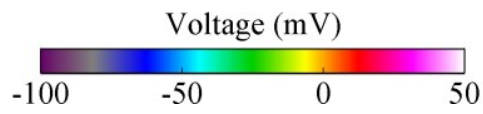

Fig. 1. Simulation set-up: a) Bipolar electrode distribution over the simulated anatomy mimicking a basket catheter. Green and blue dots indicate the measurement electrodes of each bipole, and red dots stand for the geometrical center of the bipole. Letters " $r$ " and "c" stand for row and column (spline), respectively. b-h) Snapshots of the different simulated patterns (coloured using the membrane voltage; black dots indicate the location of the bipoles centres): b) Single activation source, c) double activation source, d) anatomical reentry, e) stable functional reentry, f) unstable functional reentry, g) atrial fibrillation and h) atrial fibrillation with focal source. 
1) Single activation source (Fig. 1(b)): Activation comes from a single source located at the sphere pole, firing at a period of $350 \mathrm{~ms}$ with random jitters of $6 \mathrm{~ms}$.

2) Double activation source (Fig. 1(c)): Activation comes from two sources located at the two poles of the sphere. The two sources fire with a different initial phase and present different firing periods. The source at the top fires for the first time at $135 \mathrm{~ms}$, and it subsequently activates with a period of $350 \mathrm{~ms}$ with random jitters of 6 $\mathrm{ms}$, while the source at the bottom initially fires at $0 \mathrm{~ms}$, with a period of $360 \mathrm{~ms}$ with random jitters of $6 \mathrm{~ms}$.

3) Anatomical reentry (Fig. 1(d)): Reentry anchored to an anatomical obstacle with revolution period of $\sim 360 \mathrm{~ms}$ located at the top of the sphere combined with a functional reentry pattern (spiral) located at the bottom of the sphere.

4) Stable functional reentry (Fig. 1(e)): Two stable functional reentries (spirals) with revolution period of $\sim 200$ ms located at each pole of the sphere.

5) Unstable functional reentry (Fig. 1(f)): Unstable meandering spirals with irregular behaviour.

6) Atrial fibrillation (Fig. 1(g)): Complex fibrillation pattern with spiral breakups and multiple wavelets.

7) Atrial fibrillation with focal source (Fig. 1(h)): Combination of a complex fibrillation pattern with a localized source firing at $275 \mathrm{~ms}$, located at a pole of the sphere, which entrains the surrounding tissue.

Ionic and diffusion model parameters were modulated to reproduce the different simulation scenarios. Specifically, remodelled versions of the Courtemanche model were used in scenarios 4-5 [37] and 6-7 [36]. Conduction was assumed to be uniform and isotropic, with the diffusion coefficient $D$ ranging between 0.2 and $0.5 \mathrm{~cm}^{2} / \mathrm{s}$. ODE-PDE system integration was performed by a fully-adaptive multi-resolution algorithm [38]. Reaction and diffusion were integrated with time step $\Delta \mathrm{t}=0.1 \mathrm{~ms}$, using the Rush-Larsen non-standard finite difference forward Euler method and explicit node-centered finite difference stencils [39].

\section{B. Clinical mapping data}

Mapping data were obtained from a database of selected patients with paroxysmal AF displaying different spatiotemporal patterns of atrial organization [40]. Recordings were acquired using a Constellation "basket" catheter (Boston Scientific Inc., Natick, MA, USA) placed in the right atrium (RA). The basket catheter consisted of eight splines, each carrying eight equally spaced electrodes with $4 \mathrm{~mm}$ inter-electrode distance. Therefore, thirty-two bipolar EGMs, formed by coupling adjacent pairs of electrodes, were acquired at $1 \mathrm{kHz}$ sampling frequency and filtered between 30-500 Hz (CardioLab System, Prucka Engineering Inc., Houston, TX, USA).

Recordings had different signal lengths depending on the electrophysiological study. Therefore, for analysis purposes, a $10 \mathrm{~s}$ length signal excerpt was selected as the one maximizing the global root mean square (RMS) value of the EGM after band-pass filtering between $40-250 \mathrm{~Hz}$. This criterion aims to select a time window with (overall) good electrode contact with the atrium and to objectivize further analysis.

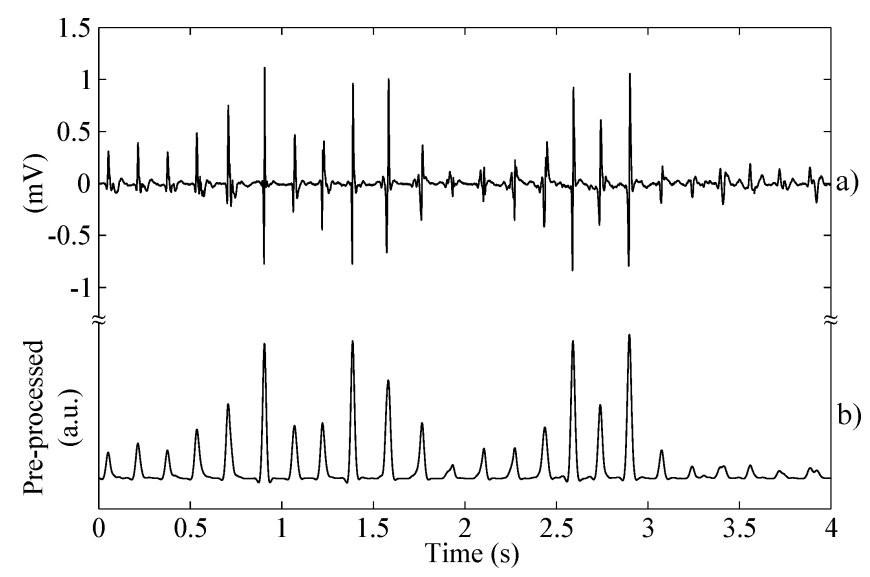

Fig. 2. EGM signal pre-processing: a) Real EGM signal, b) pre-processed EGM signal using the Botteron and Smith pre-processing chain [21].

\section{METHODS}

\section{A. MVAR signal modelling}

In this work, we considered $L$-dimensional multi-variate stochastic processes $S=\left\{S_{1}, \ldots, S_{L}\right\}$ describing the activity of different electrodes on the atria. Each set of $L$ simultaneous observations $\mathbf{s}(n)=\left[s_{1}(n), \ldots, s_{L}(n)\right]^{\mathrm{T}}$ is assumed to be represented by an MVAR model of order $m$ :

$$
\mathbf{s}(n)=\sum_{k=1}^{m} \mathbf{A}(k) \mathbf{s}(n-k)+\mathbf{v}(n),
$$

where each $\mathbf{A}(k)$ is an $L \times L$ matrix whose elements are the auto-regressive coefficients $a_{i j}(k), \quad, i, j=1 \ldots L \quad, \quad$ and $\mathbf{v}(n)=\left[v_{1}(n), \ldots, v_{L}(n)\right]^{\mathrm{T}}$ is a multi-variate white noise process defined by its covariance matrix $\Sigma_{\mathbf{v}}$.

For a given observation $\mathbf{s}(n)$, the MVAR coefficient matrices $\mathbf{A}(k)$ were estimated using the least-squares method [41] and the model order $m$ was chosen in the range $1-15$ as the value minimizing the Bayesian information criterion (BIC) [42]. In case the BIC did not reach a minimum, the model order $m$ was chosen as the first one whose successive difference in BIC is smaller than the $5 \%$ of the largest successive difference.

\section{B. Signal pre-processing}

Bipolar EGM signals were pre-processed using the Botteron and Smith pre-processing chain [21]:

1) Band-pass filtering between 40 and $250 \mathrm{~Hz}$, using a second order Butterworth IIR filter.

2) Signal rectification.

3) Low-pass filtering with $25 \mathrm{~Hz}$ cut-off frequency, using a second order Butterworth IIR filter.

This pre-processing chain aims to enhance the rhythmic properties of atrial EGM signals, simplifying their shape variations while reducing noise, as illustrated in Fig. 2. Additionally, the pre-processed signals were downsampled to 100 $\mathrm{Hz}$ in order to provide sufficient information for the MVAR 
modelling while avoiding high model orders due to data redundancy.

\section{Granger causality and predictability measures}

This section introduces the analysis framework proposed in this study to characterize different aspects of atrial activity. The framework is based on the concept of GC [34], [43], and the constituent measures are taken from a set of previous works in which they were defined to study different aspects of the dynamical dependencies of multivariate time series [44]-[46].

The GC is a measure of predictability and precedence [47]. In a bivariate context where $L=2$ processes are considered, i.e. $S=\{X, Y\}$, the source process $X$ is said to be cause of the target process $Y$ (in the sense of the GC) if the past of $X$ contains information that helps to predict the future of $Y$ over and above the information already contained in the past of $Y$ [34].

Let $x^{\prime}=x(n)$ denote the present value of a realization of the process $X$, let $\mathbf{x}^{-}=[x(n-m), \ldots, x(n-1)]^{\mathrm{T}}$ denote the vector containing the $m$ past values of $X$, and extend this notation to every considered process. Additionally, let $\sigma(A)$ be the variance of the process $A$ and $\sigma(A \mid B)$ be the residual variance of the regression of the process $A$ over the multi-variate process $B$. The residual variance was obtained from the parameters of the MVAR representation (1) using the method described in [48], which is based on the auto-covariance sequence of the MVAR process inferred from the estimated model parameters.

Then, the GC from the source process $X$ to the target process $Y$ can be written as [43], [46], [47], [49]:

$$
\begin{aligned}
G_{X \rightarrow Y} & =\ln \left(\frac{\sigma\left(y^{\prime} \mid \mathbf{y}^{-}\right)}{\sigma\left(y^{\prime} \mid \mathbf{y}^{-}, \mathbf{x}^{-}\right)}\right) \\
& =\ln \left(\sigma\left(y^{\prime} \mid \mathbf{y}^{-}\right)\right)-\ln \left(\sigma\left(y^{\prime} \mid \mathbf{y}^{-}, \mathbf{x}^{-}\right)\right)
\end{aligned}
$$

In this study, we consider the extension of the above concept to the multi-variate case [43], [47] assuming $L=3$, so that the observed process is $S=\{X, Y, Z\}$, where $Y$ is the target process and $\{X, Z\}$ are considered as source processes. In the extended framework, the predictability $P_{Y}$ of the target process $Y$ is defined as [45], [46]:

$$
P_{Y}=\ln \left(\sigma\left(y^{\prime}\right)\right)-\ln \left(\sigma\left(y^{\prime} \mid \mathbf{y}^{-}, \mathbf{x}^{-}, \mathbf{z}^{-}\right)\right),
$$

measuring how much the present of the target process $Y$ can be predicted from the knowledge of its own past and from the past of the other considered processes.

The predictability (3) can be decomposed into two terms as follows [46]:

$$
P_{Y}=S_{Y}+G_{X Z \rightarrow Y},
$$

where the first term represents the self-predictability of the process $Y$, quantifying how much of the uncertainty about the present of $Y$ can be predicted just from its own past:

$$
S_{Y}=\ln \left(\sigma\left(y^{\prime}\right)\right)-\ln \left(\sigma\left(y^{\prime} \mid \mathbf{y}^{-}\right)\right),
$$

and the second term represents the joint Granger causality, quantifying the remaining amount of uncertainty about the present of $Y$ that could not be predicted by its past but is predicted by the past of $X$ and $Z$ :

$$
G_{X Z \rightarrow Y}=\ln \left(\sigma\left(y^{\prime} \mid \mathbf{y}^{-}\right)\right)-\ln \left(\sigma\left(y^{\prime} \mid \mathbf{y}^{-}, \mathbf{x}^{-}, \mathbf{z}^{-}\right)\right) .
$$

Moreover, $G_{X Z \rightarrow Y}$ can be further decomposed following (4)-(6) [48] by either regressing first using the process $X$ or $Z$, equivalently:

$$
G_{X Z \rightarrow Y}=G_{X \rightarrow Y}+G_{Z \rightarrow \gamma \mid X}=G_{Z \rightarrow Y}+G_{X \rightarrow Y \mid Z},
$$

where $G_{X \rightarrow Y}$ and $G_{Z \rightarrow Y}$ stand for the Granger causality from $X$ and $Z$ to $Y$, respectively and obtained following (2). The terms $G_{Z \rightarrow Y X}$ and $G_{X \rightarrow Y Z}$ stand for the conditional Granger causality [43], [47] from $Z$ to $Y$ conditioned to the past of $X$, and from $X$ to $Y$ conditioned to the past of $Z$, respectively:

$$
\begin{aligned}
& G_{Z \rightarrow Y \mid X}=\ln \left(\sigma\left(y^{\prime} \mid \mathbf{y}^{-}, \mathbf{x}^{-}\right)\right)-\ln \left(\sigma\left(y^{\prime} \mid \mathbf{y}^{-}, \mathbf{x}^{-}, \mathbf{z}^{-}\right)\right), \\
& G_{X \rightarrow Y \mid Z}=\ln \left(\sigma\left(y^{\prime} \mid \mathbf{y}^{-}, \mathbf{z}^{-}\right)\right)-\ln \left(\sigma\left(y^{\prime} \mid \mathbf{y}^{-}, \mathbf{x}^{-}, \mathbf{z}^{-}\right)\right) .
\end{aligned}
$$

An alternative decomposition to (4) is [45]:

$$
P_{Y}=C_{X Z \rightarrow Y}+G_{Y \mid X Z},
$$

where the first term stands for the joint cross predictability of $Y$ from both processes $X$ and $Z$, quantifying the amount of uncertainty about the present of $Y$ that can be predicted solely from the past of $X$ and $Z$ taken together:

$$
C_{X Z \rightarrow Y}=\ln \left(\sigma\left(y^{\prime}\right)\right)-\ln \left(\sigma\left(y^{\prime} \mid \mathbf{x}^{-}, \mathbf{z}^{-}\right)\right),
$$

and the second term quantifies the concept of Granger autonomy of $Y$, measuring the improvement in the prediction of the target process $Y$ yielded by the inclusion of its own past over and above the predictability already achieved from the past of $X$ and $Z$ [44]:

$$
G_{Y \mid X Z}=\ln \left(\sigma\left(y^{\prime} \mid \mathbf{x}^{-}, \mathbf{z}^{-}\right)\right)-\ln \left(\sigma\left(y^{\prime} \mid \mathbf{y}^{-}, \mathbf{x}^{-}, \mathbf{z}^{-}\right)\right) .
$$

In the same manner as the decomposition presented in (7), $C_{X Z \rightarrow Y}$ can be decomposed as:

$$
C_{X Z \rightarrow Y}=C_{X \rightarrow Y}+C_{Z \rightarrow \gamma \mid X}=C_{Z \rightarrow Y}+C_{X \rightarrow Y Z}
$$

where $C_{X \rightarrow Y}$ and $C_{Z \rightarrow Y}$ stand for the cross predictability of $Y$ from $X$ or $Z$, respectively, and terms $C_{Z \rightarrow Y \mid X}$ and $C_{X \rightarrow Y \mid Z}$ stand for the conditional cross predictability of $Y$ from $Z$ and $X$ conditioned to $X$ and $Z$, respectively. 


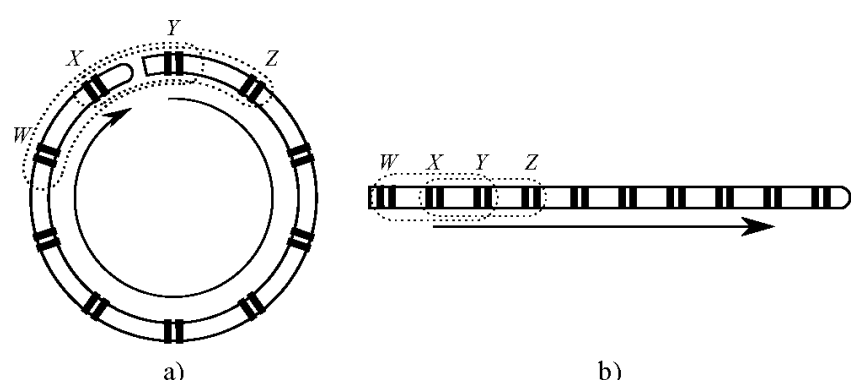

Fig. 3. Schematic of application of the three-electrode analysis scheme proposed in this work: a) over a circular catheter and b) over a linear catheter. Arrows indicate the movement of the electrode triplet for analysis.

\section{Measurement framework}

This section describes the implementation of the measures introduced in section III.C in the context of atrial activity analysis, and provides newly defined measures that were designed to highlight relevant features of interacting signals within this specific context. In order to apply the definitions formulated in section III.C, we assume that the neighbour electrodes convey the largest amount of the information relevant to the activity sensed by the target electrode under analysis. Accordingly, we propose a three-electrode analysis scheme that is applicable to any multi-electrode catheter, and is based on defining the following three processes:

1) Process $Y$ : Target electrode EGM signal.

2) Process $X$ : Leftwards neighbour electrode EGM signal.

3) Process $Z$ : Rightwards neighbour electrode EGM signal.

This three-electrode analysis scheme was shifted across the catheter electrode distribution under analysis until its complete coverage was achieved, as illustrated in Fig. 3.

All the measures introduced in section III. $C$ are related with the predictability of the signal under study (process $Y$ ) mediated by the influence of the past of the neighbour electrodes (processes $X$ and $Z$ ) and/or its own past. The aim of this work is to use these interactions to assess cardiac activity; therefore, we provide the following interpretations for some of the derived measures:

- The predictability $P_{Y}$ can be interpreted as a general measure of the regularity of the target EGM signal $Y$ that takes into account the possible influences from the neighbour EGM signals $X$ and $Z$.

- The self-predictability $S_{Y}$ and the Granger autonomy $G_{Y \backslash X Z}$ can be interpreted as measures of the local regularity of the target EGM signal $Y$, which arises from the knowledge of its own dynamics, including or not the possible effect of the neighbour EGM signals $X$ and $Z$.

- The conditional Granger causality $G_{X \rightarrow Y \mid Z}$ and $G_{Z \rightarrow Y \mid X}$ assess the information transfer from the adjacent EGM signals $X$ or $Z$ to the target electrode EGM signal $Y$, after removing the effect of the other adjacent site.

Moreover, we define the neighbour connectivity ratio $N_{Y}$ as the relative amount of information carried by the target signal that can be predicted solely from the past of the neighbour processes. This concept is quantified combining autonomy and predictability as follows:

$$
N_{Y}=1-\frac{G_{Y \backslash X Z}}{P_{Y}} .
$$

so that (13) spans from 0 to 1 , where lower values show high contribution of the autonomy in predictability, and vice versa. Note that the neighbour connectivity ratio can be formulated equivalently as $N_{Y}=C_{X Z \rightarrow Y} / P_{Y}$, which reveals that $N_{Y}$ reflects how much site $Y$ is connected with sites $X$ and $Z$, with low values indicating isolation of the target electrode since its activity is predicted mainly by its own dynamics (i.e., autonomy) but not from the dynamics of the adjacent electrodes.

After computing the complete set of GC measures across the catheter electrode distribution, we define the propagation direction as:

$$
D_{X \leftrightarrow Y}=\frac{G_{Y \rightarrow X \mid W}-G_{X \rightarrow Y \mid Z}}{G_{Y \rightarrow X \mid W}+G_{X \rightarrow Y \mid Z}},
$$

where the process $W$ stands for the adjacent electrode to $X$ in the opposite (leftwards) direction, if it is available (see Fig. 3). The propagation direction $D_{X \leftrightarrow Y}$ quantifies the dominant direction of the information transfer, i.e. the relative strength of the information transferred in one direction with respect to the information transferred in the opposite direction. This measure spans from -1 to 1 , where negative values indicate a dominant information transfer from $X$ to $Y$ and vice versa. Thus, $D_{X \leftrightarrow Y}$ can be used to track the propagation of the electrical activity throughout the catheter electrode distribution.

\section{E. Application of the framework on basket catheter data}

The measurement framework introduced in section III.D was applied on basket catheter data considering two different configurations: along the catheter splines (spline-wise analysis), or along the electrodes located at each row of the catheter in circular distribution (row-wise analysis). As the introduced measures are dependent on the considered neighbour electrodes, the analysis generally yields different measures when performed on the two configurations.

Then, activity maps that illustrate the electrical activity measured by the basket catheter were constructed combining the neighbour connectivity ratio $N_{Y}$ and the propagation direction $D_{X \leftrightarrow Y}$. In these maps, each electrode of the basket catheter was represented as a node coloured according to the value of $N_{Y}$, and pairs of adjacent electrodes were connected according to the values of $D_{X \leftrightarrow Y}$. While $D_{X \leftrightarrow Y}$ could be computed and visualized for both spline- and row-wise analyses, the visualization of $N_{Y}$ computed through spline- and row-wise analysis is not straightforward. In this study, we chose to display in the activity maps the values of $N_{Y}$ analyzed in the direction (row-wise or spline-wise) where the map has a greater average predictability (denoted as $\bar{P}_{Y}^{\mathrm{s}}$ and $\bar{P}_{Y}^{\mathrm{r}}$ for spline- and row-wise analysis, respectively). 
a)

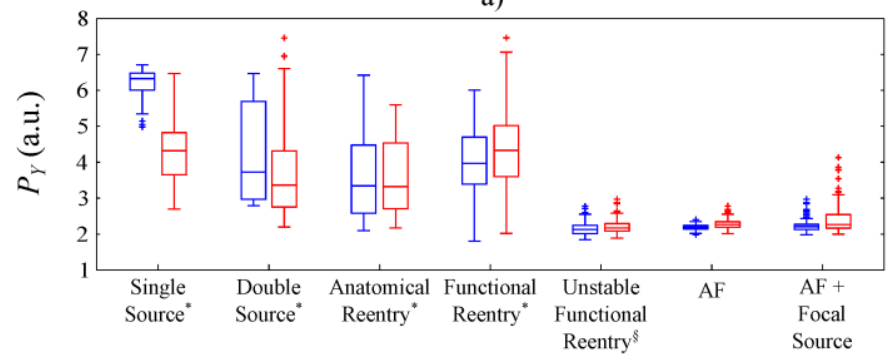

c)

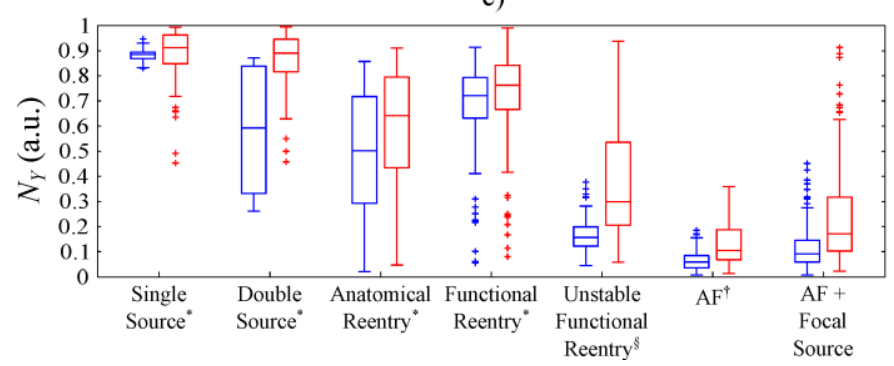

b)

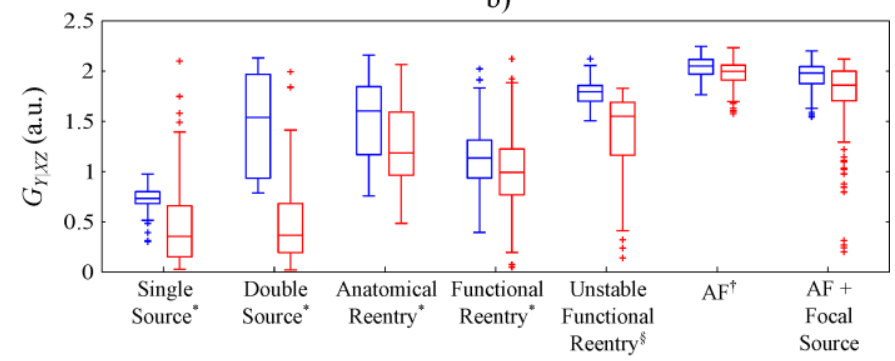

d)

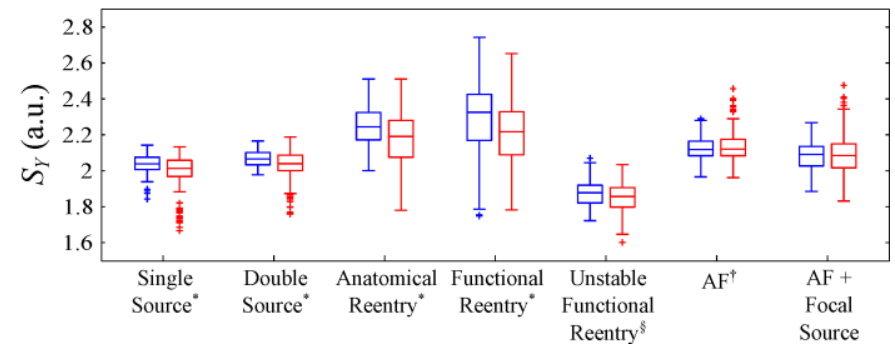

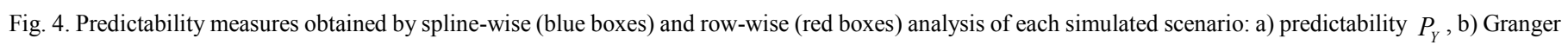

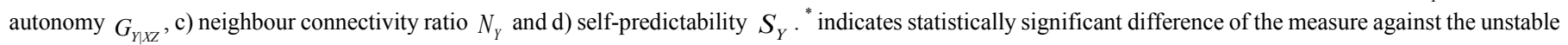
functional reentry, $\mathrm{AF}$ and $\mathrm{AF}$ with focal source scenarios $(p \leq 0.05),{ }^{\S}$ indicates statistically significant difference of the measure against $\mathrm{AF}$ and $\mathrm{AF}$ with focal source scenarios $(p \leq 0.05)$ and ${ }^{\dagger}$ indicates statistically significant difference of the measure against AF with focal source scenario $(p \leq 0.05)$.

\section{F. Statistical analysis}

The distribution of the studied GC-based measures is shown as median with the respective quartiles. The statistical significance of the differences between GC-based measures obtained from pairs of simulated scenarios is assessed, when indicated, using the Wilcoxon's test. Moreover, the statistical significance of any GC-based measure is tested against the null hypothesis of zero GC using the test described in [47]. In all statistical tests, a $p$-value $\leq 0.05$ was set as threshold for rejecting the null hypothesis.

\section{RESULTS}

\section{A. Atrial simulation analysis}

1) Predictability analysis: Figure 4 shows the distributions of the measures of predictability, self-predictability, Granger autonomy and neighbour connectivity ratio, computed for the seven simulated scenarios.

Figure 4(a) illustrates that the predictability presents the highest values for the single source scenario, and its values decrease as the simulated patterns become more complex. On the other hand, the opposite behaviour is illustrated in Fig. 4(b) for the Granger autonomy. This suggests that more regular atrial rhythms exhibit high predictability mostly related to the influence of adjacent sites on the target EGM signal. However, such influence vanishes in more complex atrial activity where a significant amount of regularity is due to local activity.

The neighbour connectivity ratio, being related to the ratio of the other two, emphasizes their relation in normalized units, reflecting the regularity of the patterns resulting from non-isolated atrial activity (Fig. 4(c)).

The self-predictability does not seem to characterize well the complexity of the simulated patterns, as it shows similar ranges for the different simulation scenarios with no clear trend with the complexity of the simulated activity (see Fig 4(d)).

As illustrated in Fig. 4, all the studied measures are able to distinguish between organized and unorganized simulated activity $(p \leq 0.05)$ in both spline-wise and row-wise configuration analysis.

2) Causality analysis: Figure 5 exemplifies the capability of the proposed framework to track the propagation of atrial activity through the propagation direction $D_{X \leftrightarrow Y}$.

Figure 5(a)-(b) illustrates the activity sensed by one of the linear splines of the simulated basket catheter in presence of a single activation source pattern and a double activation source pattern, respectively. In Fig. 5(a) activation comes from the top of the spline and propagates downwards, whereas in Fig. 5 (b) activation comes from both the top and bottom part of the spline and fuses between electrodes 4 and 5 as illustrated in the collision EGM indicated with an asterisk.

Figure 5(c)-(d) illustrates the activity sensed by electrode row \#1 in presence of an anatomical reentry and a stable functional reentry, respectively. In Fig. 5(c), the propagation follows a sequence that indicates a wavefront circulating in the direction of the circular electrode configuration. Figure 5(d) shows that electrode 18 is the earliest activated (marked with an asterisk), which suggests the presence of a source of activation at that site. Moreover, the activation linearly propagates across the studied circular configuration, ending at electrode 9 .

3) Activity mapping: Figure 6 illustrates the activity maps of the full simulated basket catheter.

The activity map for the single source scenario is shown in Fig. 6(a). This map was obtained by spline-wise computation of $N_{Y}\left(\bar{P}_{Y}^{\mathrm{s}}=6.22, \bar{P}_{Y}^{\mathrm{r}}=4.28\right)$. It shows that the wave travels 
a)
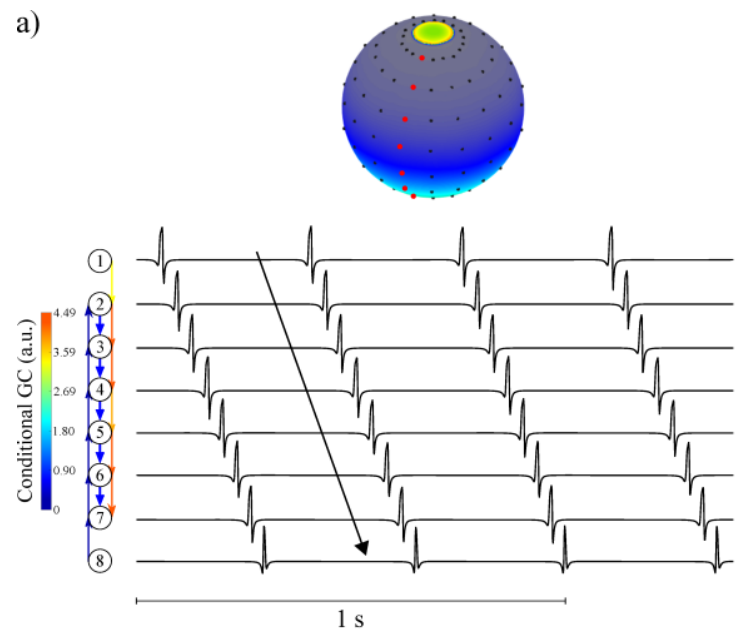

b)
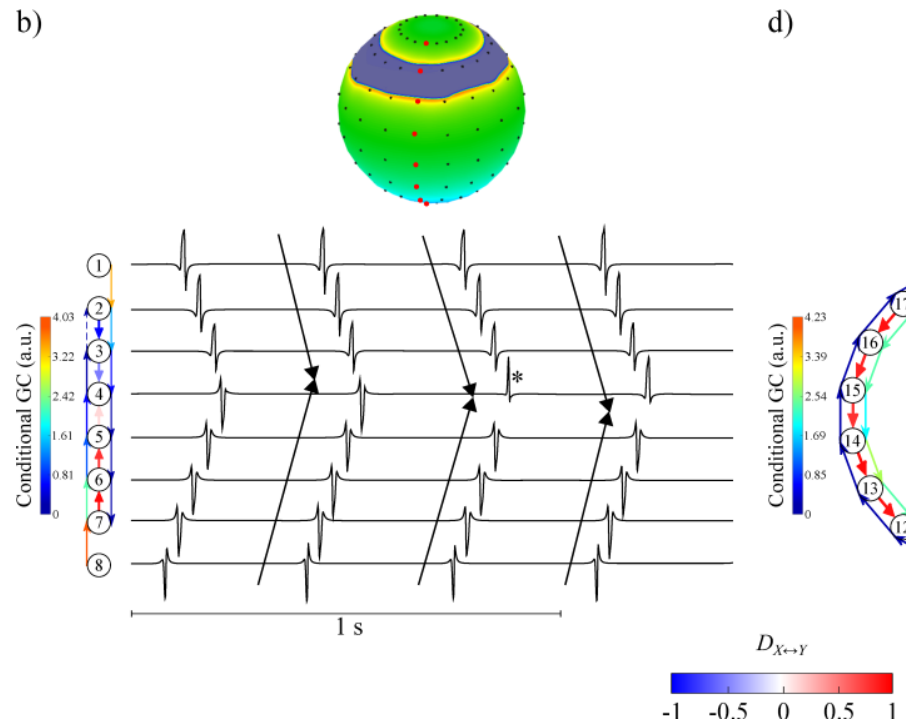

c)

d)
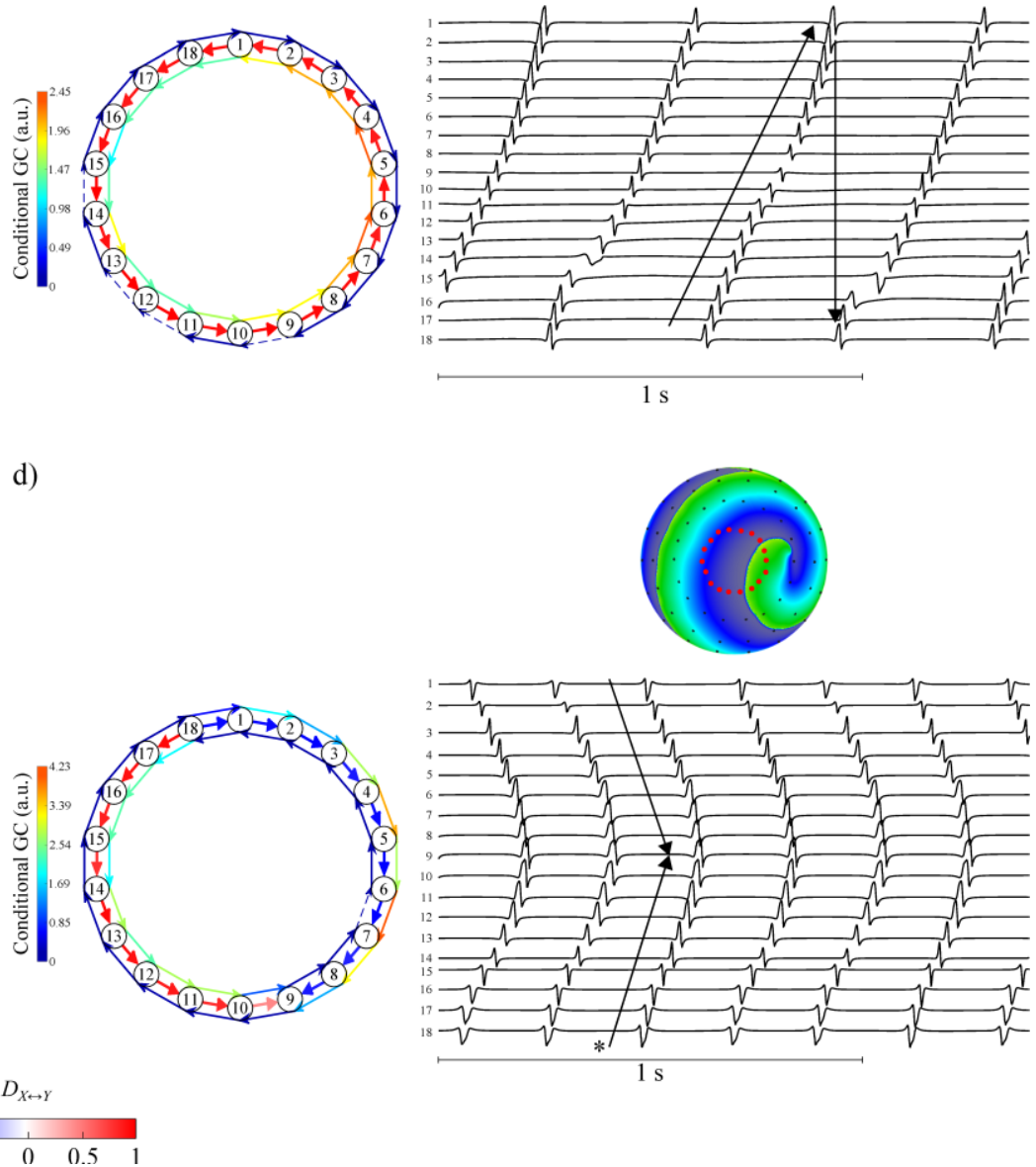
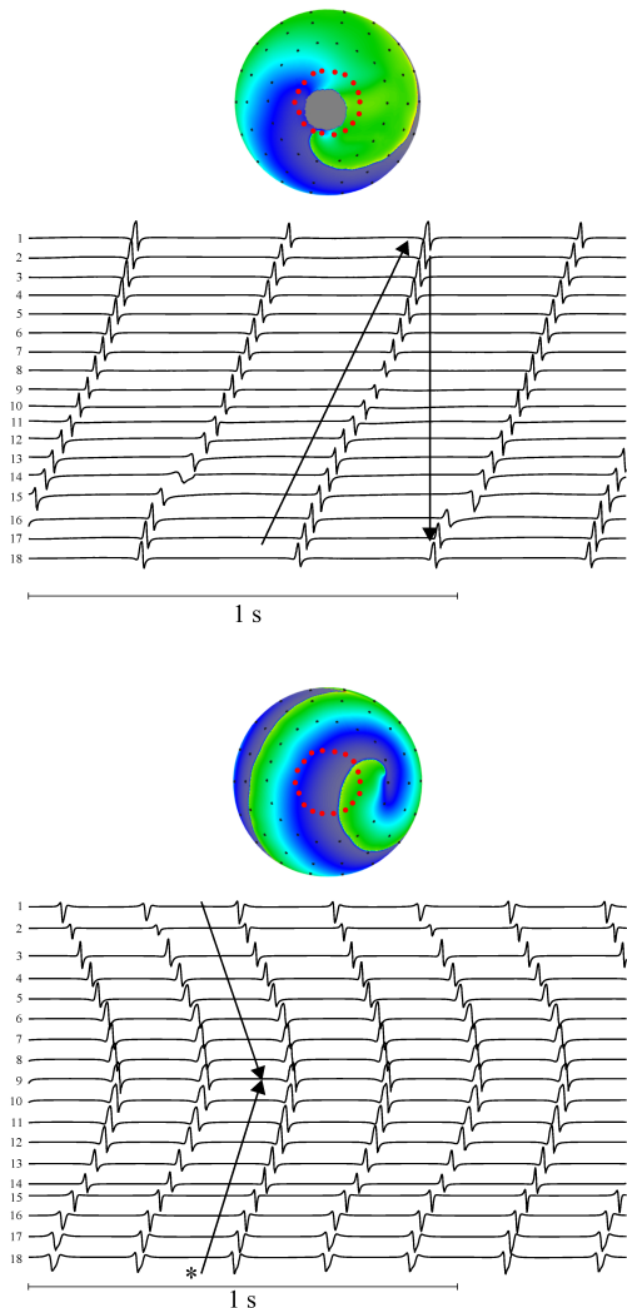

$1 \mathrm{~s}$

Fig. 5. Examples of causality analysis maps obtained using the proposed framework during simulation of: a) single source (electrode spline \#1), b) double source (electrode spline \#1), c) anatomical reentry (electrode row \#1) and d) stable functional reentry (electrode row \#1). Each panel shows: at the top, a snapshot of the simulated pattern (red dots: electrodes selected for catheter analysis); at the bottom left, a schematic of the catheter used for the causality analysis with electrode connections (numbered circles: corresponding catheter electrodes; lateral coloured arrows: conditional Granger causality, dashed if p-value $\leq 0.05$; central tick arrows: propagation direction $D_{X \leftrightarrow Y}$ ); at the bottom right, the corresponding EGM signals with propagation indicated by black arrows. The meaning of the asterisks in b) and d) is explained in section IV.A 2).

from top to bottom in a very regular fashion indicated by consistently high $D_{X \leftrightarrow Y}$ and $N_{Y}$ values. The activity map for the double source scenario is shown in Fig. 6(b). This map was obtained by spline-wise computation of $N_{Y}\left(\bar{P}_{Y}^{\mathrm{s}}=4.25, \bar{P}_{Y}^{\mathrm{r}}=\right.$ 3.68). Both the sign of the propagation direction $D_{X \leftrightarrow Y}$ and the high values of $N_{Y}$ indicate that the activation comes from each extreme of the catheter. Moreover, $N_{Y}$ values decrease towards the collision area at the center part of the catheter.

Figure 6(c) shows the activity map of the anatomical reentry scenario. This map was obtained by spline-wise computation of $N_{Y}\left(\bar{P}_{Y}^{\mathrm{s}}=3.63, \bar{P}_{Y}^{\mathrm{r}}=3.60\right)$. The different values of $N_{Y}$ and $D_{X \leftrightarrow Y}$ observed between the top and bottom parts of the catheter suggest the presence of a stable and regular pattern located at the top, with revolution direction going from spline 18 to 1 and the presence of a more unstable pattern at the bottom.
Figure 6(d) shows the activity map of the functional reentry simulation scenario. This map was obtained by row-wise computation of $N_{Y}\left(\bar{P}_{Y}^{\mathrm{s}}=3.98, \bar{P}_{Y}^{\mathrm{r}}=4.29\right)$. The low $N_{\mathrm{Y}}$ values suggest two sources of instability, the first located around the top part of splines 1-18 and the second around the bottom part of splines $2-3$. These two sources of instability are surrounded by a stable propagation observed throughout the rest of the catheter.

The activity map of the unstable functional reentry simulation scenario is shown in Fig. 6(e). This map was obtained by row-wise computation of $N_{Y}\left(\bar{P}_{Y}^{\mathrm{s}}=2.14, \bar{P}_{Y}^{\mathrm{r}}=2.21\right)$. The homogeneously low values of $N_{Y}$ suggest a high complexity of the activation throughout the catheter, related to low predictability and/or high autonomy of the atrial activity. However, a reentrant path is illustrated around splines 7-13. A video of this simulation scenario illustrating this reentrant 
a)

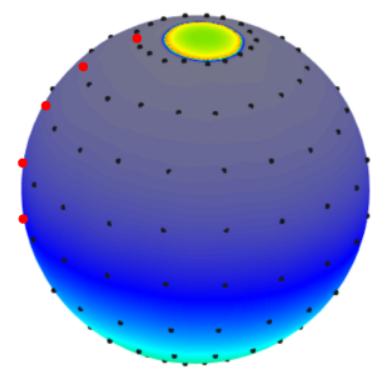

c)

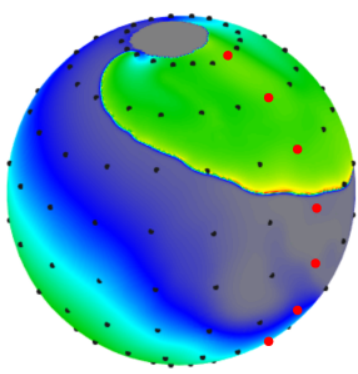

e)

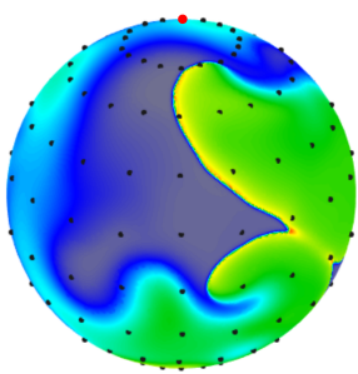

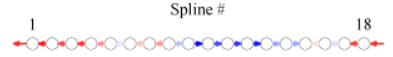

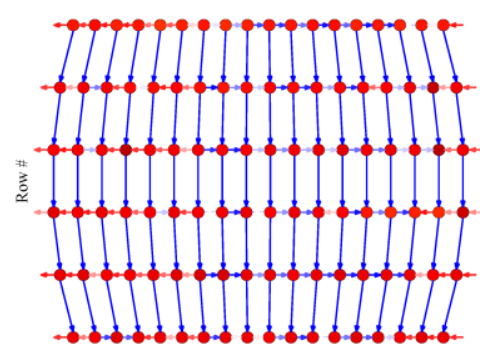

b)

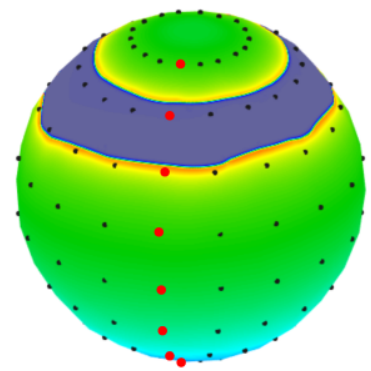

d)

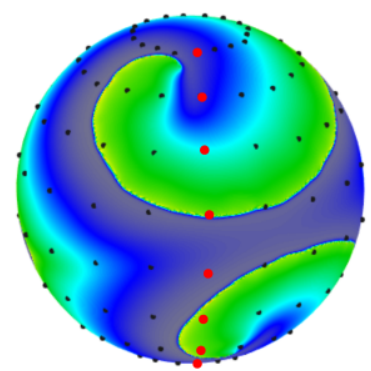

f)

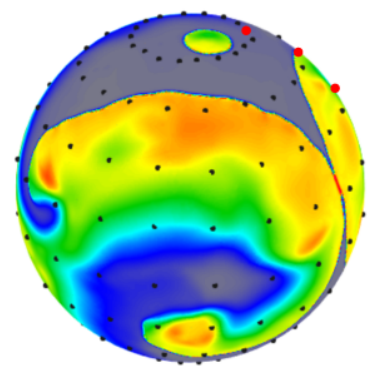

$N_{Y}$

$\begin{array}{llll}0 & 0.25 & 0.5 & 0.75\end{array}$
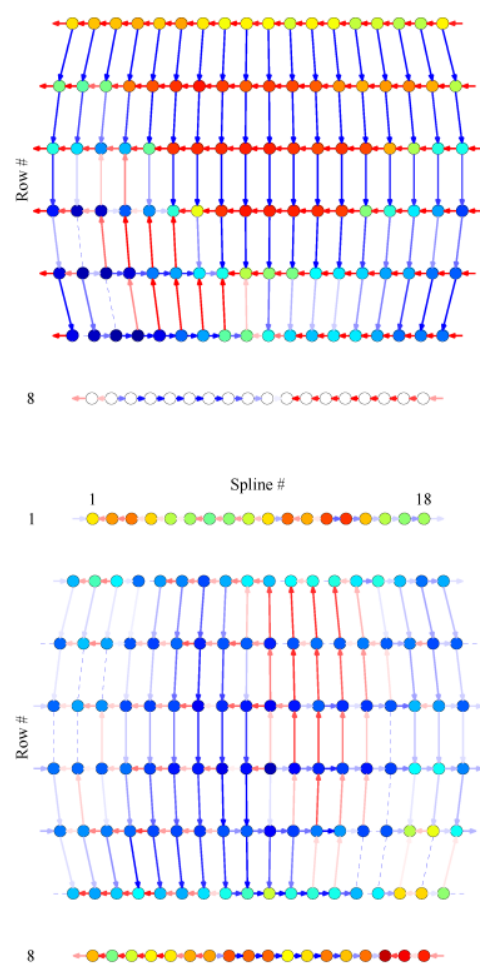

$1 \quad 1 \quad$ Spline \# 18

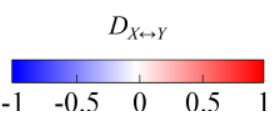

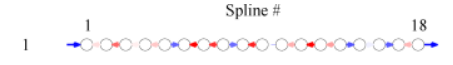

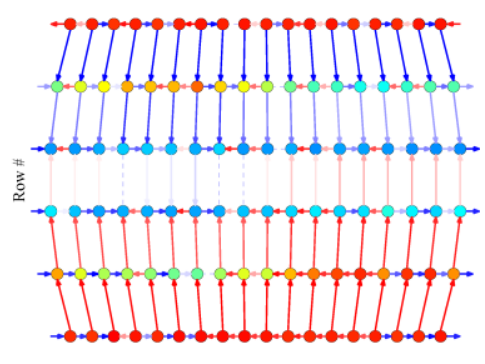

Spline
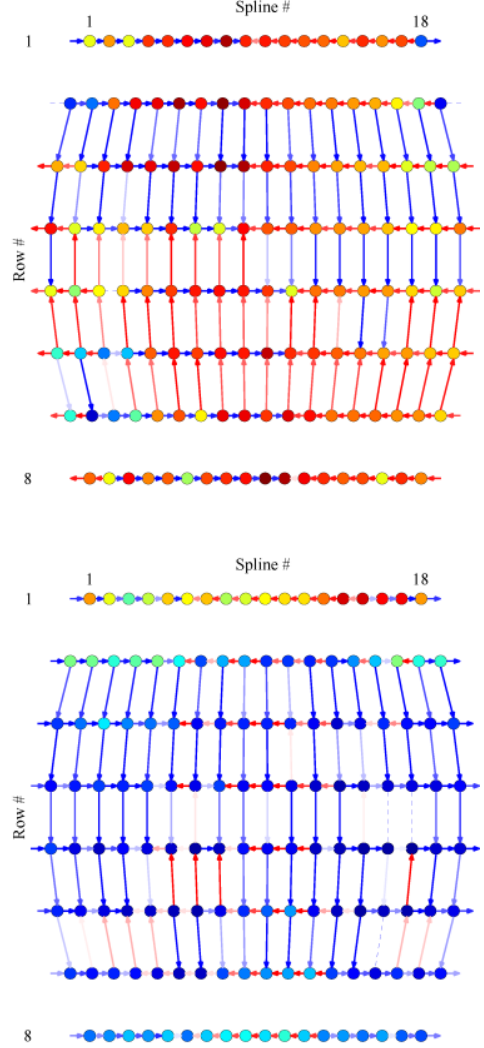

Fig. 6. Activity maps of the simulated basket catheter for simulations of: a) single source, b) double source, c) anatomical reentry, d) stable functional reentry, e) unstable functional reentry and f) AF with focal source. Each panel shows: at the left, a snapshot of the simulated scenario (red dots indicate the electrode spline \#1 with counterclockwise spline numbering) and at the right, the corresponding schematic of the simulated basket catheter whose electrodes are represented by nodes coloured corresponding to the values of $N_{Y}$ (non-coloured nodes: $N_{\mathrm{Y}}$ could not be obtained) and connected following the values of the propagation direction $D_{X \leftrightarrow Y}$ (dashed connections: no dominant direction, i.e., $\left|D_{X \leftrightarrow Y}\right|<0.05$ ). $N_{Y}$ is computed through spline-wise analysis in panels (a)-(c) and through row-wise analysis in panels (d)-(f). Note that due to geometry constraints, connections between rows $1-2$ and rows 7-8 were not possible to obtain.

path is included in the on-line supplementary material (video 1).

The activity map of the AF with focal source scenario is shown in Fig. 6(f). This map was obtained by row-wise computation of $N_{Y}\left(\bar{P}_{Y}^{\mathrm{s}}=2.24, \bar{P}_{Y}^{\mathrm{r}}=2.39\right)$. A predominant up-to-bottom propagation is identified with low $N_{Y}$ values for almost all electrode location except for those upper rows where a source of regularity is present. Moreover, high values of $N_{Y}$ are shown for splines 13-17 suggesting a displacement of the regularity source slightly towards this direction, as can be seen in the on-line supplementary material (video 2).

\section{B. Mapping data analysis}

EGM signals, acquired using a basket catheter, were studied from two patients with paroxysmal AF, showing different spatiotemporal organization patterns. In particular, two com- 
a)
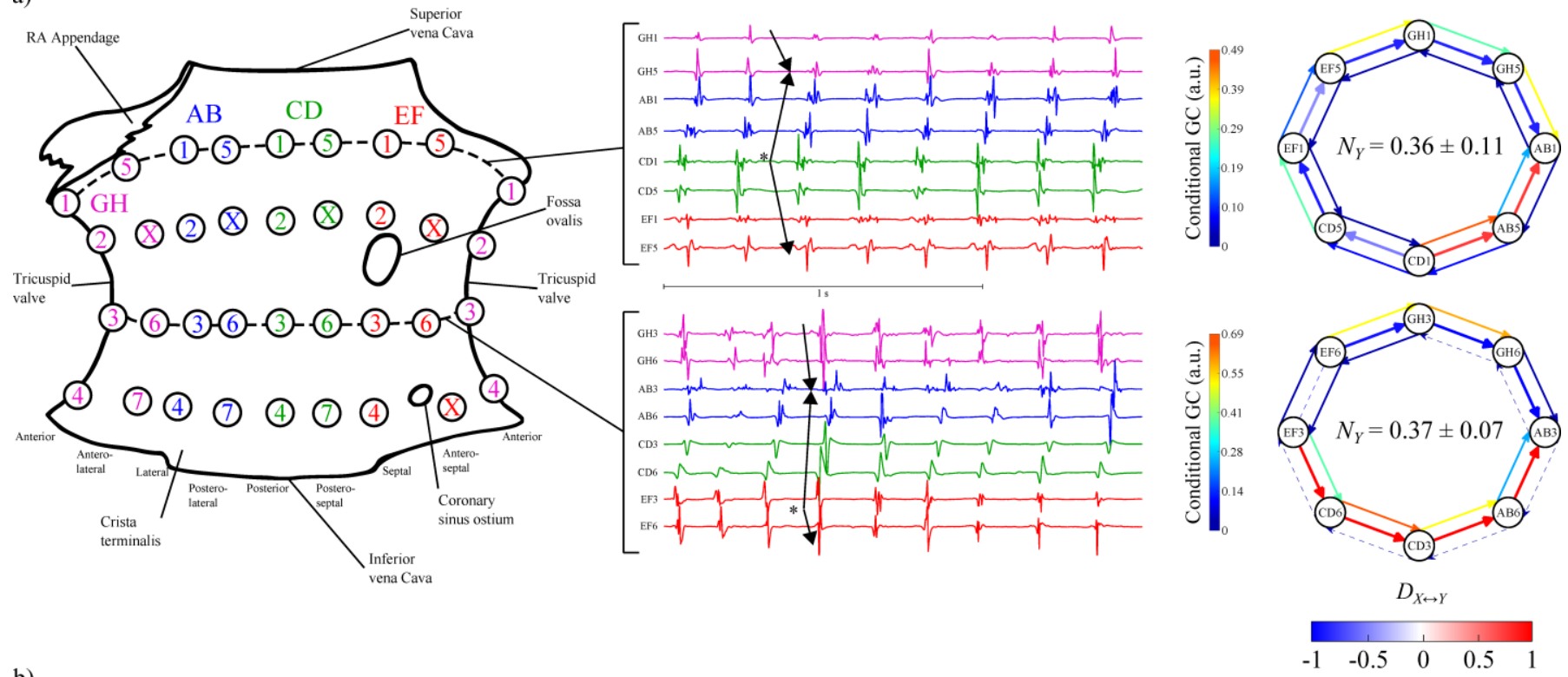

b)
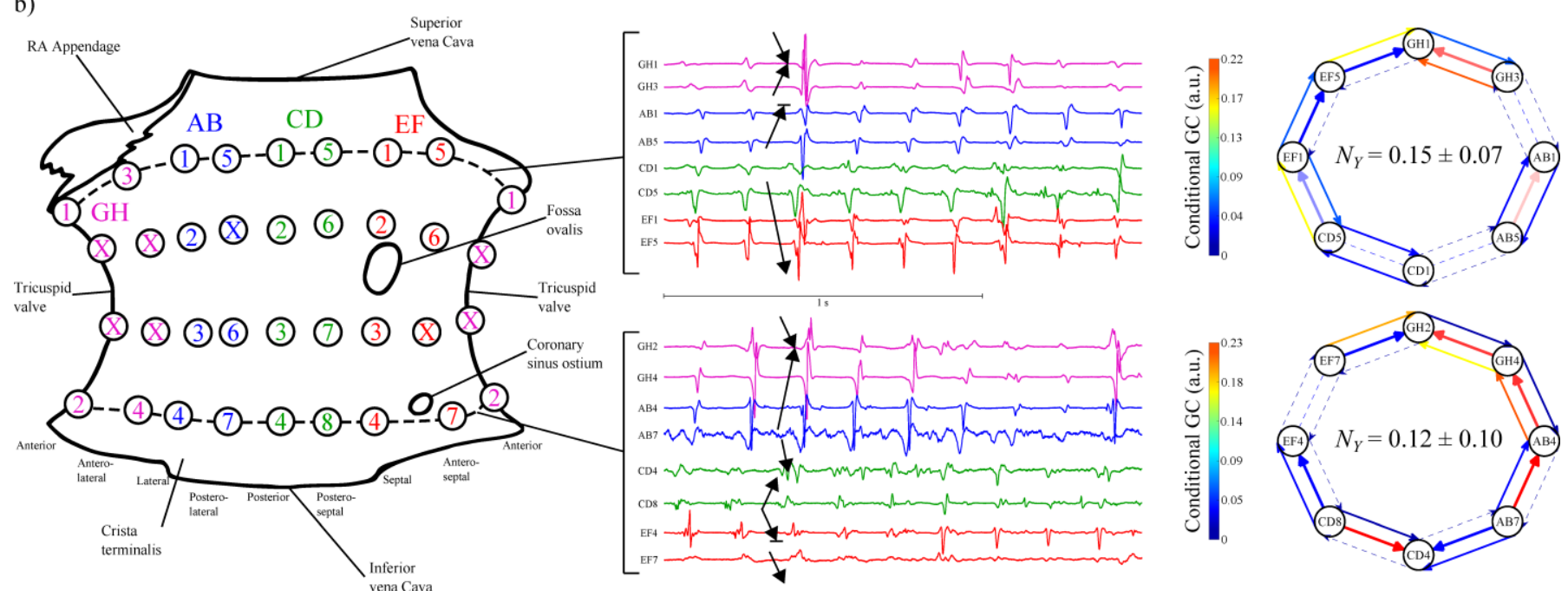

Fig. 7. Application of the proposed framework to RA basket mapping data in two patients with spatiotemporal AF patterns exhibiting different levels of complexity. Each panel shows: at the left, a schematic representation of the open RA with the position of the recording electrodes (unavailable electrodes due to miss-contact are indicated with X; electrode connected with a dashed line indicate the analyzed circular configuration); at the center, the corresponding EGM signals of the analyzed circular configuration; at the right, the causality map (named circles: corresponding catheter electrodes; lateral coloured arrows: conditional Granger causality, dashed if p-value $\leq 0.05$; central tick arrows: propagation direction $D_{X \leftrightarrow Y}$, dashed if no dominant direction is present, i.e., $\left.\left|D_{X \leftrightarrow Y}\right|<0.05\right)$ including at the centre

the average \pm SD value of $N_{Y}$. The meaning of the asterisks in a) is explained in section IV.B.

plete circular locations of the basket catheter from each patient were analysed.

In Fig. 7(a) the proposed framework tracks the complete activity, suggesting an early propagation from the posterior RA wall (electrode CD1 indicated by an asterisk) at the cranial loop that shifts towards the RA septum (electrodes EF3-6 indicated by an asterisk) at the medial loop. In Fig. 7(b), the framework identifies some electrode connections (CD5 to $\mathrm{GH} 1$ for the cranial loop and $\mathrm{AB} 7$ to $\mathrm{GH} 2$ in the caudal loop) even in the presence of a more complex propagation pattern, characterized by multiple wavefront blocks

The values of the neighbour connectivity ratio obtained for the two circular electrode configurations of Fig. 7(a) (presented as mean \pm standard deviation (SD) over all electrodes) indicate a degree of regularity higher than that observed for the circular configurations of Fig. 7(b). These higher values are in agreement with the clear propagation observed in Fig. 7 (a) looking at the causality analysis maps.

\section{DISCUSSION}

This paper introduces a framework, based on the concept of GC, to quantify the predictability of intracardiac signals assessing both the regularity of the activity of individual cardiac sites and the interactions among spatially separated sites. The GC is a measure of prediction and precedence, which is assessed in this study within the context of a linear analysis framework. Accordingly, MVAR models of the pre-processed bipolar EGMs were used for computing the GC-related measures based on the residuals variance of different process regressions [47], [49]. 
In this work, GC analysis is formalized for atrial signals in a unified framework by providing a set of prediction measures, which are used and interpreted within the physiology of AF. Note that not all GC-based measurements presented in section III. $C$ are relevant for the aim of this work (e.g. the conditioned decompositions of the joint cross-predictability), but they have been defined to achieve completeness in the presentation of the predictability framework.

The work extends several methodologies already proposed in this context [28]-[33]. For instance, frequency domain causality analysis of AF was performed in [28], [29] via partial directed coherence (PDC). Compared to PDC, our approach provides causality measures that are easier to interpret, because our method does not require identifying a frequency band to analyze interactions. Moreover, our approach is more comprehensive as it explores a wide range of dynamical properties of the atrial signals besides causality, such as overall and self-predictability, propagation direction and neighbourhood connectivity. On the other hand, the approaches in [30]-[33] also proposed time-domain GC-based measures, but are limited in that they define bi-variate measures, which are sensitive to spurious connectivity induced by a common driver or cascade effects of non-modelled signals. Our framework implements a multivariate formulation of GC measures that allows a more precise identification of propagation patterns. It may take any number of neighbouring atrial sites into account, just by considering $Z$ as a multi-variate process that includes all the $L-2$ remaining neighbour electrodes under analysis.

In addition to the multi-variate formulation, the proposed framework extends measures of spatial organization such as cross-correlation or cross-spectral measures (proposed, e.g., in [13], [14], [21]) also by providing the important information about the direction of the interactions, which is closely related to the direction of atrial wave propagation. Also, compared with activation sequence-based approaches (e.g. in [16]-[20]), the main advantage of the proposed framework is that the activation detection step is not necessary and, therefore, the analysis outcomes are not limited by the activation detection accuracy during complex activity.

With the aim of providing a detailed view of the potential usefulness of the methodology, the evaluation of the proposed framework was conducted using both simulations of different AF mechanisms and clinical mapping data. The simulation study employed seven different simulation scenarios, covering a wide range of conditions of atrial patterns from regular to irregular activity. Moreover, the derived EGM signals were obtained mimicking a basket catheter configuration, which provides a more realistic situation found in an electrophysiology lab. This basket catheter configuration allowed also to test the framework employing different electrode distributions (i.e., circular and linear) and to prove the generality of the proposed three-electrode analysis scheme.

\section{A. Quantification of the regularity of simulated atrial activity}

The predictability and its conditioned decompositions, the self-predictability and the Granger autonomy, allow to measure different aspects of the regularity of the signal and the underlying activity. We found that predictability and Granger autonomy display an opposite behaviour with respect to the complexity of the simulated activity. On the other hand, the self-predictability does not show a clear trend that differentiates regular from irregular rhythms, in contrast with Granger autonomy, as illustrated in Fig. 4. This raises the importance of spatiotemporal analysis because the self-predictability was mainly affected by the surrounding signals in organized or regular rhythms and this effect was removed by definition in the computation of the Granger autonomy measure.

The neighbour connectivity ratio combines predictability and Granger autonomy, providing a normalized descriptive measure of the atrial rhythms that accounts for both the global regularity and the degree of connectivity. The low values of the neighbour connectivity ratio found for the unstable simulated scenarios (unstable functional reentry, AF and AF with focal source), compared to the organized scenarios ( $p$-value $<$ 0.05 for all comparisons) reflect the ability of this measure to quantify isolation. Therefore, the more irregular the propagation is, the less predictable the signal is from the adjacent electrodes, and lower values are attained for the neighbour connectivity ratio.

All these measures are dependent on the direction of the propagation with respect to the orientation of the three-electrode analysis scheme used for rhythm analysis and characterization (see Fig. 4). Important differences appear in scenarios where the propagation direction follows the direction of one of the two electrode distributions (i.e., spline-wise or row-wise). In particular, single/double source scenarios present high/low values during row-wise analysis. In these cases, the activation wavefront passes simultaneously through all electrodes, allowing the adjacent electrodes to explain the activity of the target electrode. On the other hand, spline-wise analysis of anatomical reentry and functional reentry show lower regularity, compared with row-wise analysis, due to the differential behaviours of each hemisphere and the global analysis.

\section{B. Activity tracking capability}

The analysis of the conditional Granger causality by means of the propagation direction $D_{X \leftrightarrow Y}$ illustrates the sequence of activation sensed by the catheter. The sign and the values of the propagation direction quantify the predominant direction and the relative strength of information transfer between two electrodes and track the propagation through the catheter, as is illustrated in simulations in Figs. 5-6. Moreover, Fig. 7 shows examples of the application of the proposed methodology, when atrial activity was mapped using the propagation direction in paroxysmal AF patients showing different organization patterns. The good tracking capability observed in these exemplary cases suggests the usefulness of the proposed measure of propagation direction.

Methodologically the propagation direction is defined using the conditional Granger causality $G_{X \rightarrow Y \mid Z}$ and $G_{Y \rightarrow X \mid W}$ in order to isolate the interaction between electrodes $X$ and $Y$ from its other neighbours ( $W$ and $Z$, respectively). In the case of $L=3, D_{X \leftrightarrow Y}$ could have been equivalently defined in terms of the Granger causalities between $X$ and $Y$ (i.e. $G_{X \rightarrow Y}$ and $\left.G_{Y \rightarrow X}\right)$. However, the latter definition would be limited within an extended framework with $L>3$, where additional condi- 
tioning terms would be needed to avoid over-estimation of the existing interactions between close neighbour electrodes with the target electrode.

Moreover, combining the propagation direction $D_{X \leftrightarrow Y}$ and the neighbour connectivity ratio $N_{Y}$, it is possible to obtain comprehensive activity maps that include, within the same picture, information about the direction of propagation and the areas of stability of such propagation (see Fig. 6). These maps allow to observe the underlying activity in detail. For instance, Fig. 6(a) shows a stable map reflecting the presence of a single source of activation, while Fig. 6(b) shows decreasing connectivity values while approaching the central area of the sphere, where wavefronts from the two sources collide. This last result reflects the instability of the collision, and explains the wide range of regularity observed in Fig. 4. Figure 6(c) shows different behaviour between hemispheres, with an organized propagation at the top of the sphere due to the anatomical reentry and less organized propagation at the bottom due to the collision of the "tail" and the "head" of the reentrant wavefront. Figure 6(d) shows medium-to-high regularity in the whole catheter except for those locations where the tips of the functional reentries (spirals) are localized. Figure 6(e) shows a preferential reentry path from top-to-bottom around splines 6-9 and from bottom-to-top around splines 9-13, whereas unstable patterns occur in the rest of the simulated sphere. This is illustrated in the slow motion video included in the on-line supplementary material (video 1), which is coherent with the activity map of Fig. 6(e). Finally, Fig. 6(f) shows that the top of the sphere, where the focal source of activation entrains the tissue during AF, is the most regular part of the simulation scenario. This agrees with what is expected and can be observed in the on-line supplementary material (video 2).

While the activity maps have been obtained in this work following the analysis direction that maximizes the average predictability value, both spline- and row-wise analysis are needed for their construction. Therefore, although this maximization criterion can be considered as a guideline for default visualization if the framework was applied to clinical practice, it is flexible enough to allow displaying the results of both analyses. Such flexibility extends also to the proposed three-electrode analysis scheme, which can be expanded to include more than two neighbours to match the analysis to a particular multi-electrode catheter.

\section{Limitations of the framework}

The proposed framework characterizes atrial activity by implementing a linear MVAR modelling analysis of the dynamics and interactions between nearby cardiac sites. While the presence of nonlinear dynamics may limit the descriptive capability of the framework, we showed that the linear assumption appears to be sufficient to track most of the activity in both simulations and clinical mapping data. Nevertheless, it may be interesting to consider extensions of our approach to nonlinear models, although not many examples can be found in the analysis of AF intracardiac signals [50].

The proposed three-electrode analysis scheme assumes one-electrode neighbourhood and one-dimensional interaction. This topology is applicable to any multi-electrode cath- eter, but this assumption may not be always accomplished due to electrode sparsity. Other limitations of the proposed scheme are related to the number of available electrodes, especially for extremal electrodes in linear catheter configurations, and the need of achieving complete contact for all electrodes in order to perform a continuous analysis of the cardiac activity. This was especially noticed in real data analysis, which was limited by the lack of contact for some electrodes. Nevertheless, the proposed three-electrode analysis scheme aims to be general and applicable on any multi-electrode catheter without being tailored to a particular catheter type or electrode distribution. Moreover, linear and circular catheters are still the most commonly used in clinical practice. However, some of the limitations described above may be alleviated by choosing other neighbourhood electrode topologies, as allowed by the flexibility of the framework.

\section{Clinical relevance and future work}

The proposed activity maps provide a global visualization of a multi-electrode catheter that may be potentially useful for identifying fibrillation sources and guiding catheter ablation interventions. For that purpose, further evaluation is needed in additional clinical scenarios of AF.

\section{CONCLUSION}

This paper introduces a linear predictability framework for analyzing cardiac activity and interactions during AF based on GC definitions. The method provides regularity measures that can distinguish the complexity between different atrial rhythms. Moreover, it can be applied to track and map the underlying cardiac activity in any simultaneous multi-electrode catheter, not requiring activation detector or post-processing algorithms. The proposed global mapping of regularity and connectivity of the activity acquired from multi-electrode catheters, simultaneously showing signal propagation and stability, can be useful for interpreting such activity and supporting clinicians during ablation interventions.

\section{REFERENCES}

[1] G. Boriani, "The epidemiologic threat of atrial fibrillation: Need for secondary, primary, and primordial prevention," CHEST J., vol. 147, no. 1, pp. 109-119, 2015.

[2] A. J. Camm et al., "Guidelines for the management of atrial fibrillation: The task force for the management of atrial fibrillation of the European Society of Cardiology (ESC)," Eur. Heart J., vol. 31, no. 19, pp. 2369-2429, 2010.

[3] V. E. Hagens et al., "Effect of rate or rhythm control on quality of life in persistent atrial fibrillation. Results from the Rate Control Versus Electrical Cardioversion (RACE) study," J. Am. Coll. Cardiol., vol. 43, no. 2, pp. 241-247, 2004.

[4] G. Thrall et al., "Quality of life in patients with atrial fibrillation: A systematic review," Am. J. Med., vol. 119, no. 5, pp. 448.e1-e19, 2006.

[5] T. Lewis, "Auricular fibrillation: A common clinical condition," $\mathrm{Br}$. Med. J., vol. 2, p. 1528, 1909.

[6] G. K. Moe and J. A. Abildskov, "Atrial fibrillation as a self-sustaining arrhythmia independent of focal discharge," Am. Heart J., vol. 58, no. 1, pp. 59-70, 1959.

[7] M. A. Allessie et al., "Experimental evaluation of Moe's multiple wavelet hypothesis of atrial fibrillation," in Cardiac Electrophysiology and Arrhythmias, D. P. Zipes and J. Jalife, Eds. Grune \& Stratton, 1985, pp. 265-276.

[8] M. Haïssaguerre et al., "Spontaneous initiation of atrial fibrillation by ectopic beats originating in the pulmonary veins," N. Engl. J. Med., 
vol. 339 , no. 10 , pp. $659-666,1998$.

[9] J. Eckstein et al., "Mapping of atrial fibrillation - Basic research and clinical applications," Swiss Med. Wkly., vol. 139, no. 35-36, pp. 496-504, 2009.

[10] N. M. S. de Groot et al., "Electropathological substrate of longstanding persistent atrial fibrillation in patients with structural heart disease: Epicardial breakthrough," Circulation, vol. 122, no. 17, pp. 1674-1682, 2010.

[11] K. Nademanee et al., "A New approach for catheter ablation of atrial fibrillation: Mapping of the electrophysiologic substrate," J. Am. Coll. Cardiol., vol. 43, no. 11, pp. 2044-2053, 2004.

[12] K. Nademanee et al., "Catheter ablation of atrial fibrillation guided by complex fractionated atrial electrogram mapping of atrial fibrillation substrate," J. Cardiol., vol. 55, no. 1, pp. 1-12, 2010.

[13] P. Sanders et al., "Spectral analysis identifies sites of high-frequency activity maintaining atrial fibrillation in humans," Circulation, vol. 112, no. 6, pp. 789-797, 2005.

[14] T. H. Everett et al., "Frequency domain algorithm for quantifying atrial fibrillation organization to increase defibrillation efficacy," IEEE Trans. Biomed. Eng., vol. 48, no. 9, pp. 969-978, Sept. 2001.

[15] F. Ravelli and M. Masè, "Computational mapping in atrial fibrillation: How the integration of signal-derived maps may guide the localization of critical sources," Europace, vol. 16, no. 5, pp. 714-723, 2014.

[16] R. P. M. Houben et al., "Automatic mapping of human atrial fibrillation by template matching," Hear. Rhythm, vol. 3, no. 10, pp. 1221-1228, 2006.

[17] R. P. M. Houben, N. M. S. de Groot, and M. A. Allessie, "Analysis of fractionated atrial fibrillation electrograms by wavelet decomposition," IEEE Trans. Biomed. Eng., vol. 57, no. 6, pp. 1388-1398, Jun. 2010.

[18] A. Alcaine et al., "A wavelet-based activation detector for bipolar electrogram analysis during atrial fibrillation," in Proc. Computing in Cardiology, 2012, vol. 39, pp. 717-720.

[19] M. El Haddad et al., "Algorithmic detection of the beginning and end of bipolar electrograms: Implications for novel methods to assess local activation time during atrial tachycardia," Biomed. Signal Process. Control, vol. 8, no. 6, pp. 981-991, 2013.

[20] A. Alcaine et al., "Spatiotemporal model-based estimation of high-density atrial fibrillation activation maps," Digit. Signal Process., vol. 54, pp. 64-74, 2016.

[21] G. W. Botteron and J. M. Smith, "A technique for measurement of the extent of spatial organization of atrial activation during atrial fibrillation in the intact human heart," IEEE Trans. Biomed. Eng., vol. 42, no. 6, pp. 579-586, Jun. 1995.

[22] H. J. Sih et al., "A high-temporal resolution algorithm for quantifying organization during atrial fibrillation," IEEE Trans. Biomed. Eng., vol. 46, no. 4, pp. 440-450, Apr. 1999.

[23] V. Barbaro et al., "Automated classification of human atrial fibrillation from intraatrial electrograms," Pacing Clin. Electrophysiol., vol. 23, no. 2, pp. 192-202, 2000.

[24] L. Faes et al., "A method for quantifying atrial fibrillation organization based on wave-morphology similarity," IEEE Trans. Biomed. Eng., vol. 49, no. 12, pp. 1504-1513, Dec. 2002.

[25] U. Richter et al., "Right atrial organization and wavefront analysis in atrial fibrillation," Med. Biol. Eng. Comput., vol. 47, no. 12, pp. 1237-1246, 2009.

[26] F. Ravelli et al., "The logical operator map identifies novel candidate markers for critical sites in patients with atrial fibrillation," Prog. Biophys. Mol. Biol., vol. 115, no. 2-3, pp. 186-197, 2014.

[27] S. M. Narayan et al., "Computational mapping identifies localized mechanisms for ablation of atrial fibrillation," PLoS One, vol. 7, no. 9, p. e46034, 2012.

[28] U. Richter et al., "A novel approach to propagation pattern analysis in intracardiac atrial fibrillation signals," Ann. Biomed. Eng., vol. 39, no. 1, pp. 310-323, 2011.

[29] U. Richter et al., "Propagation pattern analysis during atrial fibrillation based on sparse modeling," IEEE Trans. Biomed. Eng., vol. 59, no. 5, pp. 1319-1328, May 2012.
[30] M. Rodrigo et al., "Causality relation map: A novel methodology for the identification of hierarchical fibrillatory processes," in Proc. Computing in Cardiology, 2011, vol. 38, pp. 173-176.

[31] M. Rodrigo et al., "Identification of fibrillatory sources by measuring causal relationships," in Proc. Computing in Cardiology, 2012, vol. 39, pp. 705-708.

[32] M. Rodrigo et al., "Identification of ablation sites in atrial flutter by causal method," in Proc. Computing in Cardiology, 2013, vol. 40, pp. 707-710.

[33] M. Rodrigo et al., "Identification of dominant excitation patterns and sources of atrial fibrillation by causality analysis," Ann. Biomed. Eng., vol. 44, no. 8, pp. 2364-2376, 2016.

[34] C. W. J. Granger, "Investigating causal relations by econometric models and cross-spectral methods," Econometrica, vol. 37, no. 3, pp. 424-438, 1969 .

[35] M. Courtemanche, R. J. Ramirez, and S. Nattel, "Ionic mechanisms underlying human atrial action potential properties: Insights from a mathematical model," Am. J. Physiol. - Hear. Circ. Physiol., vol. 275, no. 1, pp. H301-H321, Jul. 1998.

[36] V. Jacquemet et al., "Study of unipolar electrogram morphology in a computer model of atrial fibrillation," J. Cardiovasc. Electrophysiol., vol. 14, no. s10, pp. S172-S179, 2003.

[37] M. Courtemanche, R. J. Ramirez, and S. Nattel, "Ionic targets for drug therapy and atrial fibrillation-induced electrical remodeling: Insights from a mathematical model," Cardiovasc. Res., vol. 42, no. 2, pp. $477-489,1999$

[38] A. Cristoforetti, M. Masè, and F. Ravelli, "A fully adaptive multiresolution algorithm for atrial arrhythmia simulation on anatomically realistic unstructured meshes," IEEE Trans. Biomed. Eng., vol. 60, no. 9, pp. 2585-2593, Sept. 2013.

[39] V. Jacquemet and C. S. Henriquez, "Finite volume stiffness matrix for solving anisotropic cardiac propagation in 2-D and 3-D unstructured meshes," IEEE Trans. Biomed. Eng., vol. 52, no. 8, pp. 1490-1492, Aug. 2005.

[40] F. Ravelli et al., "Wave similarity mapping shows the spatiotemporal distribution of fibrillatory wave complexity in the human right atrium during paroxysmal and chronic atrial fibrillation," J. Cardiovasc. Electrophysiol., vol. 16, no. 10, pp. 1071-1076, 2005.

[41] H. Lütkepohl, New Introduction to Multiple Time Series Analysis. Berlin, Heidelberg: Springer Berlin Heidelberg, 2005.

[42] G. Schwarz, "Estimating the dimension of a model," Ann. Stat., vol. 6, no. 2, pp. 461-464, 1978.

[43] A. Porta and L. Faes, "Wiener-Granger causality in network physiology with applications to cardiovascular control and neuroscience," Proc. IEEE, vol. 104, no. 2, pp. 282-309, Feb. 2016.

[44] A. K. Seth, "Measuring autonomy and emergence via Granger causality," Artif. Life, vol. 16, no. 2, pp. 179-196, 2010.

[45] L. Faes, A. Porta, and G. Nollo, "Information decomposition in bivariate systems: Theory and application to cardiorespiratory dynamics," Entropy, vol. 17, no. 1, pp. 277-303, 2015.

[46] L. Faes et al., "Predictability decomposition detects the impairment of brain-heart dynamical networks during sleep disorders and their recovery with treatment," Philos. Trans. A. Math. Phys. Eng. Sci., vol. 374, no. 2067, 2016.

[47] L. Barnett and A. K. Seth, "The MVGC Multivariate Granger Causality toolbox: A new approach to Granger-causal inference," $J$. Neurosci. Methods, vol. 223, pp. 50-68, 2014.

[48] L. Faes et al., "Estimating the decomposition of predictive information in multivariate systems," Phys. Rev. E, vol. 91, no. 3, pp. 032904-1 032904-16, 2015.

[49] D. Chicharro, "On the spectral formulation of Granger causality," Biol. Cybern., vol. 105, no. 5-6, pp. 331-347, 2011.

[50] B. P. Hoekstra et al., "Non-linear time series analysis: Methods and applications to atrial fibrillation," Ann. Ist. Super. Sanita, vol. 37, no. 3, pp. 325-333, 2001. 